Voix et Images

voixetimages

\title{
Entretien avec Herménégilde Chiasson
}

Benoit Doyon-Gosselin

Volume 35, numéro 1 (103), automne 2009

Herménégilde Chiasson

URI : https://id.erudit.org/iderudit/038566ar

DOI : https://doi.org/10.7202/038566ar

Aller au sommaire du numéro

Éditeur(s)

Université du Québec à Montréal

ISSN

0318-9201 (imprimé)

1705-933X (numérique)

Découvrir la revue

Citer ce document

Doyon-Gosselin, B. (2009). Entretien avec Herménégilde Chiasson. Voix et Images, 35(1), 13-21. https://doi.org/10.7202/038566ar d'utilisation que vous pouvez consulter en ligne.

https://apropos.erudit.org/fr/usagers/politique-dutilisation/ 


\title{
ENTRETIEN AVEC HERMÉNÉGILDE CHIASSON
}

\author{
$++$ \\ BENOIT DOYON-GOSSELIN \\ Université Laval
}

VoIX ET IMAGES Après trente-cinq ans de carrière dans les domaines de la poésie, du thêâtre, du cinéma et des arts visuels, on finit par s'interroger sur leur genèse. Que retrouve-t-on à l'origine du processus créatif et de la formation de l'artiste?

HERMÉNÉGILDE CHIASSON Je dirais que ce sont les arts visuels, parce que finalement, j'ai fait la plus grande partie de mes études dans cette discipline. C'est le seul endroit où je suis vraiment à l'aise, parce que dans les autres domaines, je me sens comme un dilettante. Autrefois, j'étais très mal à l'aise quand les gens disaient: "Ah, oui, Herménégilde Chiasson, le cinéaste, ou le peintre, ou l'auteur dramatique.» Comme j'ai travaillé dans plusieurs disciplines, je me sentais toujours aliéné lorsque je me retrouvais en présence d'écrivains, mais cela se produisait aussi avec les autres disciplines. Mais dans les arts visuels, je suis dans mon élément, je perçois mieux ce qui se passe. Maintenant, de plus en plus, je vois qu'il s'agit d'une symbiose. J'ai l'impression d'avoir produit une œuvre très baroque et assez postmoderne, en tout cas très éclatée.

VoIX ET IMAGES Si on recule dans le temps, on se demande pourquoi un petit garçon de Saint-Simon au Nouveau-Brunswick, né dans les années 1940, choisit les arts visuels comme domaine d'études, et ce, jusqu'au doctorat.

HERMÉnÉGILDE CHIASSON Mes parents faisaient des choses reliées aux arts visuels, mais de manière très, très naïve. Ma mère faisait de l'artisanat, des courtepointes, des fleurs en papier. Elle fabriquait toutes sortes d'objets qui finalement étaient connexes au domaine de l'art ou de l'artisanat. J'étais vraiment fasciné par les couleurs qu'elle utilisait. Mon père, lui, dessinait. C'est assez étrange parce que la couleur et le dessin, c'est un peu ce qui compose le langage visuel. Et puis dans la maison, chez nous, il n'y avait pas de livres. Mes parents étaient plus ou moins analphabètes. Quand tu ne sais pas lire, tu n'achètes évidemment pas de livres.

voIX ET IMAGES Lorsque vous étiez étudiant, au cours des années 1970, quel rôle a joué l'Université de Moncton dans votre réflexion sur la société acadienne et sur les arts?

HERMÉNÉGILDE CHIASSON Déjà je m'apercevais, lorsque j'étais à Saint-Simon, qu'il y avait une tradition très forte, surtout en musique, et qu'il y avait aussi une présence moderne. Pendant les danses, par exemple, on voyait deux groupes: un groupe plus 
âgé qui voulait des sets carrés, des reels et de la musique au violon et les autres qui, eux, voulaient écouter de la musique rock'n'roll. Il y avait toujours une espèce de tension entre les générations. Tout ce qui avait précédé, je voulais m'en distancier et aller voir ce qui se passait ailleurs. L'université en ce sens a été un outil incroyable parce qu'elle nous a permis de sortir tous ensemble. Moi, j'ai fait deux ans - les deux dernières années - à l'Université de Moncton; les deux premières années, j'étais interne au Collège Saint-Joseph. J'ai vraiment connu les deux mondes. La différence, c'est que Moncton est une ville, une ville plutôt modeste, mais quand même une ville. À Moncton, il y avait un groupe de jeunes intellectuels, dont on voit d'ailleurs une certaine partie dans le film de Léonard Forest, Les Acadiens de la dispersion. J'étais dans le domaine de la culture, et en culture, à ce moment-là, on n'entendait pas beaucoup de discours critique, car les discours étaient surtout générés en politique et en sciences sociales. C'étaient des domaines qui m'intéressaient plus ou moins, je les trouvais relativement ennuyants. J'ai toujours eu un regard assez fataliste par rapport à la politique; j'ai l'impression que la politique se répète et que le pouvoir, un peu comme Foucault le disait, c'est le pouvoir, et cela se reproduit à l'échelle. La preuve, c'est que ceux qui étaient alors extrêmement radicaux sont devenus des gens assez conformistes par après. C'est pour cette raison que la culture a produit, selon moi, le discours le plus important et le plus pertinent; $c^{\prime}$ est là qu'on peut changer les mentalités en profondeur, et je pense que je m'en suis aperçu à ce moment-là, à l'Université de Moncton.

VoIX ET IMAGES La majorité des titres de vos recueils de poésie est formée d'un seul mot au pluriel; ce sont des titres laconiques. Comment justifiez-vous ce choix?

HERMÉNÉGILDE CHIASSON Quand j'ai commencé à écrire, les titres des recueils et surtout des poèmes étaient assez longs. Je pense à Rapport sur l'état de mes illusions. Ensuite, pendant une période de dix ans, j'ai continué d'écrire, mais je n'ai pas publié. Quand j'ai recommencé à publier, je me suis dit que j'allais désormais faire des titres d'un seul mot, des titres qui sont synthétiques, mais en même temps qui proposent des genres de résistances: un mot, ça intrigue beaucoup plus qu'une phrase. Au fond, c'est un peu comme les peintres qui au lieu de donner des titres à leurs tableaux donnent des chiffres, par exemple, Composition $n^{\circ} 22$. Le lecteur ne peut pas s'accrocher à grand-chose. Le titre intrigue et synthétise.

VoIX ET IMAGES Depuis une quinzaine d'années au moins, on trouve dans vos recueils une volonté de répertorier tout ce qui forme le quotidien...

HERMÉNÉGILDE CHIASSON Les deux premiers recueils étaient beaucoup plus politiques. Après, quand je suis revenu à la publication, l'Acadie était rendue dans une autre phase de son évolution, une période marquée par l'œuvre de Gérald Leblanc, très axée sur le quotidien. Je trouvais que c'était plus intéressant, et cela s'est poursuivi jusqu'à Béatitudes, où j'ai voulu établir un contraste entre un quotidien vraiment plat et banal et le côté un peu plus sublime de ce même quotidien qui, à un moment donné, peut se déplier et s'extraire de ces actions plutôt banales. C'est pour cette raison surtout que j'ai résisté à écrire en vers; pour moi, la poésie demeure très diffuse et existe partout. C'est une conception plutôt surréaliste. Au fond, toute ma formation en arts visuels s'est transférée en littérature. Le simple fait de répertorier des actes quotidiens, de les transcrire est un acte littéraire assez banal, mais le style 
finit par leur conférer une distinction qui les singularise et les fait sortir de leur anonymat normal.

VoIX ET IMAGES Il existe un certain déficit romanesque dans les milieux minoritaires, alors que la poésie publiée et le thêâtre joué demeurent les deux genres dominants. Pourquoi n'avez-vous jamais touché au genre romanesque?

HERMÉNÉGILDE CHIASSON Même l'œuvre de France Daigle traduit la volonté d'un long cheminement vers le roman. Je pense que ça commence, un peu comme chez Proust, par: «Je veux écrire un roman.» Cette volonté-là va finir par se déplier et éventuellement produire un roman. Tandis que chez moi, il n'y a jamais eu la volonté d'écrire un roman. Cela ne m'a jamais traversé l'esprit, sauf récemment, où je me suis dit que ce serait intéressant de produire un exercice du genre. C'est un roman épistolaire qui raconte l'histoire d'un réalisateur acadien qui veut produire un film de fiction. Les trente-trois lettres qu'il reçoit correspondent aux trente-trois chants de $L^{\prime}$ Enfer de Dante et proviennent d'un assortiment de personnages allant d'une de ses anciennes blondes à la prose terrifiante de Téléfilm Canada. Ce roman s'inscrit dans une trilogie, et je voudrais publier les trois volumes dans la même année, mais il me faudra du temps pour la compléter. J'ai terminé le premier volume lors d'une résidence d'auteur à l'Université d'Ottawa où je pouvais me concentrer uniquement sur l'écriture, mais vivant en Acadie, je suis sans cesse sollicité pour toutes sortes de projets, de rencontres, de travaux qui finissent par gruger le temps que je pourrais consacrer à des projets plus importants, comme cette trilogie. Le drame de ma vie, c'est sans doute la distraction.

voIX ET IMAGES Dans Petites difficultés d'existence, France Daigle écrit: «- C'est quoi un intellectuel, vraiment? - Ben, ça serait quelqu'un qui pense beaucoup pis qu'y le laisse à saouère. [...] - Nomme-z-en ouère... - Ben, je sais pas moi... Je croirais qu'Hermé en serait un. » Est-ce difficile d'être un intellectuel en Acadie? D'être un écrivain et un intellectuel?

HERMÉNÉGILDE CHIASSON Être intellectuel, c'est un travail très exigeant et très important. Je me souviens d'un film sur l'Afrique du Nord dans lequel quelqu'un demandait: «Comment se fait-il que le climat social s'est détérioré à ce point?» Et un des personnages du film répondait: "C'est parce que les intellectuels n'ont pas fait leur travail.» Je trouve que le discours demeure très important. C'est probablement une des raisons pour lesquelles j'ai été nommé au poste de lieutenant-gouverneur. On a dû se dire: «Oui, c'est un artiste, oui, il vient du domaine culturel, mais il peut aussi tenir un discours.» Pour revenir à votre question: est-ce difficile? Oui, parce que les intellectuels ont besoin de débattre leurs idées et ce n'est pas toujours possible. Heureusement, je ne suis pas le seul en Acadie, mais le nombre est quand même restreint, et les occasions de rencontres, assez limitées. Pour ce qui est d'être écrivain, c'est un peu la même situation. J'ai souvent l'impression d'assumer ces fonctions par défaut, en attendant.

voIX ET IMAGES Ce ne sont pas tous les artistes, évidemment, qui peuvent tenir un discours, autrement que dans leur œuvre.

HERMÉNÉGILDE CHIASSON Je me suis préparé à cela. J'ai l'impression d'avoir étudié longtemps parce que je voulais avoir un regard critique par rapport à ce que je faisais. Cela m'a toujours placé dans une marge où je n'ai pas vraiment souffert, mais une marge qui m'éloigne tout de même un peu du domaine culturel. Je souhaitais 
avoir un certain contrôle sur le discours généré à mon endroit, une certaine connaissance, de telle sorte que si on me critiquait, je pouvais voir d'où cette critique venait. Par ailleurs, j'ai toujours été très bon joueur en ce qui a trait à la critique. Je n'ai jamais tenu rancune à personne pour une mauvaise critique.

voix ET IMAGES Les Éditions d'Acadie ont fermé leurs portes en 2000. La revue Éloizes n'existe plus. Que pensez-vous de l'institution littéraire acadienne en 2009? HERMÉNÉGILDE CHIASSON Je pense que c'est très nouveau d'avoir des institutions culturelles en Acadie. Pendant très longtemps, les gens ont fonctionné de manière très artisanale. Tout d'un coup, on s'est retrouvé avec des artistes professionnels. $C^{\prime}$ est le passage du communautaire au professionnalisme qui devient compliqué. On a souvent l'impression qu'il s'agit uniquement de faire les choses, que leur qualité importe peu, que tout le monde est égal. Par exemple, on pourrait croire que quelqu'un dont l'œuvre se résume à une douzaine de tableaux a atteint le même statut qu'un peintre comme Roméo Savoie qui y a mis toute sa vie. Il y a aussi d'autres facteurs: la quasi-absence de critiques, l'omniprésence du genre poétique, pour ne citer que ceux-là. J'ai toujours cru que la poésie représentait la première parole; la deuxième serait le théâtre et la troisième, le roman. Ce sont toujours les mêmes personnes qui sont là, un peu désignées pour garder le fort. Aussi, il y a le lien entre le Québec et l'Acadie qui s'est détérioré au cours de ces dernières années, ce que je trouve vraiment malheureux. Il y eut un temps où des auteurs comme Yolande Villemaire, Claude Beausoleil ou Jean-Paul Daoust, pour ne nommer que ceux-là, faisaient partie de notre entourage. Il y a une sorte de tension qui s'est développée entre le Québec et l'Acadie. Il y a toujours eu des tensions politiques, mais je pense qu'au-delà de cela, il reste que les Québécois sont proches de nos préoccupations. Nous faisons tous partie de la francophonie américaine, et à ce titre-là nous devrions avoir des liens plus étroits et plus cordiaux.

VoIX ET IMAGES Vous avez déjà affirmé qu'il y avait peu ou pas de relève en Acadie, pas seulement en poésie mais dans d'autres domaines, notamment dans le cas des intellectuels qui expriment des idées et participent à des débats. C'est un des points centraux du désaccord qui existe avec la génération plus jeune. Que pensez-vous de la relève en littérature acadienne?

HERMÉNÉGILDE CHIASSON Ā un moment donné, je trouvais que les tenants de la jeune littérature mettaient énormément d'énergie sur la forme, sur le chiac surtout qui, selon moi, est une dimension formelle. Je pense que ce qui est important, c'est ce qu'on génère comme idées, ce qu'on enclenche comme mouvement, ce à quoi on croit. C'est René Huyghe qui disait: «Une grande œuvre d'art, c'est d'abord avoir quelque chose à dire, deuxièmement être touché par ce que l'on dit et troisièmement avoir les moyens intellectuels ou matériels pour le dire.» Je trouve que les premières œuvres d'Emma Haché ou de Georgette LeBlanc, c'est exactement ça. Ce sont des auteures qui ont vraiment voulu s'investir dans une œuvre littéraire, pas juste voir un peu comment ça feel de faire un livre [rires]. Il y a eu à mon sujet d'importantes tensions avec le milieu à Moncton où, pour un temps, j'ai été complètement marginalisé par des gens qui maintenant ne font plus grand-chose. Je trouvais que le fond du débat n'avait rien de littéraire, que tout ça gravitait autour de conflits de personnalités. J'aurais aimé faire face à des idées autres que de dire que je n'écrivais 
pas en chiac et qu'à ce titre-là je devais être exclu. S'ils avaient eu des arguments intéressants, j'aurais adoré en parler, enclencher un véritable débat. Mais finalement, c'était uniquement de l'opinion, non pas du discours mais plutôt de l'opinion. Et ça, c'est assez banal.

voix ET IMAGES Que pensez-vous de l'utilisation du chiac dans la chanson (le groupe Radio Radio), la bande dessinée (Acadieman), le roman, le théâtre et la poésie?

HERMÉNÉGILDE CHIASSON Je crois que cela a désormais une grande importance parce que de l'extérieur, il s'agit d'une forme d'exotisme. Je me dis que du moment où l'on écoute la manière dont les gens parlent, ou l'accent qu'ils ont, ou toutes autres particularités, on ne les écoute plus vraiment. C'est un peu ce que je trouve malheureux par rapport au chiac: les gens ne vont pas écouter le discours, ils vont seulement être fascinés, charmés, séduits par des artifices, par des effets. Je pense que la littérature, $c^{\prime}$ est autre chose et je déplore le fait qu'on soit toujours pris avec des problèmes d'oralité, de passage de l'oral à l'écrit. J'ai beaucoup de respect pour les gens qui n'ont pas eu le choix, mais pour ceux qui l'ont, j'ai un peu plus de réticences. Il faut dire aussi que le chiac n'est pas vraiment dans ma réalité puisque je viens de la péninsule acadienne, une région souvent prise à partie par les Chiacs pour son français plus près du français standard. De l'extérieur, je comprends qu'on puisse l'utiliser effectivement - c'est un matériau absolument fascinant et désormais très étudié. Quand je regarde Acadieman, je trouve que c'est vraiment drôle, et aussi Radio Radio. Je trouve qu'il y a une musicalité dans cette créolisation du langage, mais dans un rapport artistique et culturel. Lorsqu'on en fait le symbole d'une affirmation sociale, je trouve que c'est assez limité et, je dois dire, assez désolant.

voix ET IMAGes Parlons un peu de votre rapport à l'Acadie. Est-ce qu'il s'agit d'une relation amour-haine? Vous défendez beaucoup l'Acadie face au Québec et avez choisi d'y rester pour qu'une société acadienne moderne puisse prendre forme sur son territoire, mais vous êtes extrêmement critique par rapport à elle, à son attachement au passé, au folklore, aux traditions. Est-ce que ces contradictions servent de stimulant pour votre création?

hermÉnÉGILDE CHIAsson Probablement, mais c'est un peu aussi le phénomène du clan, c'est-à-dire que lorsque je suis à l'intérieur, j'ai évidemment un regard critique par rapport à ce qu'on pourrait faire, par rapport à toutes les faiblesses et les lacunes. Quand je suis à l'extérieur, tout à coup, je fais partie de ce clan et lorsque ce dernier est attaqué, indépendamment d'où vient l'attaque, il faut résister.

VoIX ET IMAGES Vous vous êtes indigné pendant longtemps de l'hégémonie culturelle du Québec qui gère les institutions selon sa propre vision de la francophonie canadienne. Voyez-vous à ce titre des améliorations ou est-ce le statu quo?

HERMÉnÉGILDE CHIASSON Les institutions québécoises, je les trouve merveilleuses. Mais quand on parle de Radio-Canada, qui est une institution fédérale à laquelle nous contribuons tous, ou du Conseil des arts du Canada, en d'autres mots des institutions où je vois qu'il y a vraiment une disparité de représentation et de distribution du financement, c'est là qu'il y a un problème. Finalement, il y a toujours eu un lien intellectuel, émotif avec le Québec. En ce qui concerne les institutions politiques, pour moi, le fait de dire «Radio-Canada» constitue une usurpation d'une appellation, qu'il faudrait modifier. 
voIX ET IMAGES Où en est l'Acadie aujourd'hui ? Les bilans que vous proposez périodiquement sont toujours très sombres, comme si l'Acadie reculait au lieu d'avancer. Est-ce que l'Acadie est une cause perdue?

HERMÉNÉGILDE CHIASSON J'ai toujours eu un regard assez sombre sur l'Acadie, ce que Gérald Leblanc m’a toujours reproché. Je lui disais: «Je suis réaliste, Gérald! Je ne suis pas pessimiste, je suis réaliste!» Mais je pense qu'il y a eu une effervescence dans les années 1970: la découverte, à cause de l'Université de Moncton, et une ouverture sur le monde par les médias. Puis, tout d'un coup, il y a eu un certain recul, une individualisation. C'est un phénomène qui n'est pas nécessairement acadien. Je trouve que les arts sont probablement un des domaines les plus importants qui soient parce qu'on peut convaincre les gens, on peut reformuler la question, on peut souvent donner des éléments de réponse qui vont faire que la société en général va pouvoir être touchée. Je pense qu'on ne passe à l'action que lorsqu'on est touché au plan émotif. Intellectuellement, on peut comprendre, mais il y a un monde entre savoir et croire. J'ai l'impression que dans les années 1970, il y a eu cette effervescence surprenante, alors que maintenant on est revenu à une position mitoyenne, et je ne sais pas exactement ce qui va advenir ensuite. Au départ, on était tellement surpris de nous-mêmes, d'être Acadiens, et à l'extérieur aussi, car les Acadiens avaient jusqu'alors été vus comme des gens très timorés, très silencieux, craintifs, historiquement absents, neutres et tout d'un coup, il y avait cette exubérance. Après ça, c'est comme si on s'était dit que l'Acadie pouvait fonctionner toute seule. Par exemple, durant la Convention d'orientation nationale des années 1970, on a voté une province acadienne; les gens sont partis chez eux et ils ont cru que ça allait se faire tout seul. Il y a des gens qui mettent le drapeau acadien sur le mât le 15 août, qui ont leur plaque, leur autocollant acadien sur leur pare-chocs et pour eux, cela suffit. Le reste va se faire tout seul.

voIX ET IMAGES Est-ce plus facile ou plus difficile d'être un artiste venant d'un petit milieu qui lutte pour sa survie?

HERMÉNÉGILDE CHIASSON Il est sûr que c'est plus facile, parce qu'on obtient une notoriété assez rapidement. Dans mon cas, cela m'a vraiment surpris. Une journée j'étais quelqu'un qui marchait sur la rue Saint-Georges, anonyme, et le lendemain, j'avais des articles dans Le Devoir et La Presse. Cela m'a donné une espèce de vertige et c'est l'une des raisons pour lesquelles je n'ai pas publié pendant dix ans. Tu te dis: «Wow, Mourir à Scoudouc, ça a vraiment été majeur, maintenant, qu'est-ce que je peux faire?»

voIX et IMAGEs Comme Bonheur d'occasion pour Gabrielle Roy : d'une certaine façon, la difficulté après, c'est de trouver sa voie, de penser à écrire d'autres grands romans ou à faire d'autres recueils aussi puissants.

HERMÉNÉGILDE CHIASSON Exactement. J'ai eu de la difficulté avant d'adopter une attitude plutôt zen et de dire: «Aujourd'hui, c'est le mieux que je peux faire, demain, je ferai autre chose. Ça ne sera pas mieux, pas pire, mais autre chose.» Au moment où j'ai pu le faire, j'ai pu recommencer à publier. Et pour revenir un peu à la question minoritaire, je dirais que c'est facile de commencer: on publie un livre, on est un écrivain. Je me demande si ce n'est pas Antonine Maillet qui disait: «Le premier livre, ce n'est pas grave, on ne sait pas ce qu'on fait. Le deuxième, là, on essaye de 
reproduire le premier et d'habitude, c'est plus ambigu, et c'est au troisième, je crois, qu'on devient vraiment un écrivain.» $C^{\prime}$ est à ce moment-là que le travail commence et qu'on en fait un quatrième, un cinquième, un sixième.

voIX ET IMAGES La longévité n'est pas donnée à tout le monde. Si on prend les trois œuvres majeures de la poésie acadienne moderne, on constate que vous être le seul écrivain de longue haleine; on dira ce qu'on voudra, Raymond Guy LeBlanc, c'est l'écrivain d'un seul recueil, Cri de terre, tout comme Guy Arsenault (Acadie Rock), alors que vous vous maintenez depuis trente-cinq ans dans différents domaines.

HERMÉNÉGILDE CHIASSON Il y a le fait aussi que dans un milieu comme celui dans lequel je travaille, on est appelé à faire beaucoup de choses. Roméo Savoie, le peintre, me disait un jour: "Tu sais, on a tout fait, on a fait les galeries, on a fait les œuvres, on s'est critiqué, on s'est collectionné, on a été le public. » J'ai l'impression que la même chose s'est produite en littérature. Finalement, on est comme une escouade. Un temps, je connaissais tout le monde qui allait venir au vernissage, qui allait venir au thêâtre, alors que maintenant, je vais au thêâtre et il y a plein de gens que je ne connais pas, et ça me rend un peu nerveux. Je me dis que dans un milieu majoritaire, ça doit être merveilleux de seulement être écrivain. Tu amènes le manuscrit à l'éditeur et il fait le reste.

voIX ET IMAGES Est-ce que la mondialisation des échanges et de la culture change quelque chose pour une petite culture comme l'Acadie? La question pour les artistes de partir ou rester en Acadie se pose-t-elle de la même façon à l'ère de la mondialisation?

HERMÉNÉGILDE CHIASSON Il est évident qu'à l'heure actuelle, il y a une plus grande ouverture. À l'époque où j'ai publié mon premier livre, Mourir à Scoudouc, j'avais été invité à la Rencontre internationale des écrivains québécois ici, à Québec. J'avais demandé qui serait là et on m'avait dit qu'il y aurait Julio Cortázar. Moi, Cortázar, j'avais lu un texte de lui, ça m'avait renversé et je m'étais dit: "Qu'est-ce que je vais aller faire, moi, pauvre petit Acadien, à Québec? Qu'est-ce que je vais leur dire? » Je n'ai jamais voulu être dans cette position-là, entendre quelqu'un dire: «Ah, eh bien, c'est un Acadien, vous comprenez, ils viennent de loin, ils parlent drôlement. » Alors j'avais prétexté je ne sais trop quoi pour ne pas y aller. À ce moment-là, c'était tellement nouveau, on était tellement conscients de notre identité, de notre marginalité, de notre présence. Je suis allé à la Rencontre internationale depuis, mais beaucoup plus tard... Ce que je trouve intéressant à l'heure actuelle, c'est que les jeunes artistes acadiens sortent; ils vont partout, ils n'ont aucun complexe. Je trouve qu'en ce sens-là, c'est une amélioration. Et pour les gens de ma génération aussi, il y a des acquis. Mais évidemment, le danger, c'est d'être complètement absorbé par cette masse.

VoIX ET IMAGES En même temps, il reste que l'Acadie se construit sur un territoire. On peut écrire de partout, mais si on veut participer à la société acadienne dans son plein sens...

HERMÉNÉGILDE CHIASSON Oui, je suis assez critique face aux gens qui sont à l'extérieur de l'Acadie. Je n'ai rien contre le fait que les gens quittent ou qu'ils continuent à faire une œuvre ailleurs, mais lorsqu'à un moment donné, ils prennent la parole et s'expriment en spécialistes, alors que ça fait des années qu'ils ne sont pas là, je me 
dis: «Si jamais je quittais l'Acadie, je ne sais pas, j'écrirais sur autre chose, je ferais autre chose.» Évidemment, nous, on est en Acadie, et j'écris de plus en plus rarement sur l'Acadie. En poésie, tout au moins, c'est pratiquement absent, mais c'est là quand même puisqu'on ne peut pas échapper à sa réalité. Alors, ma manière d'écrire, ma manière de faire des phrases, tout ça, ça reste vraiment acadien, mais c'est sûrement plus inconscient qu'apparent.

VoIX ET IMAGES Est-ce qu'un artiste très attaché à la défense de son milieu particulier et qui tient à lui donner une voix aspire aussi à l'universel?

HERMÉNÉGILDE CHIASSON Je dis souvent que je situe mon lecteur en Afrique. Quand je suis allé à Dakar, j'ai lu mes poèmes, et je n'ai pas eu à leur donner un lexique; ils m'ont très bien compris et ça s'est bien passé. Donc, c'est un peu ce que j'entends lorsque je dis, et je le dis souvent, qu'on a le bonheur de fonctionner dans un code qui est lu par deux cents millions de personnes. C'est merveilleux. Si j'étais un écrivain suédois, je serais lu par six millions de personnes; pour les autres, il faudrait que je sois traduit. C'est pour cette raison qu'il est intéressant d'écrire dans ce code-là, de maîtriser ce code-là. Quand on lance un livre, il s'en va comme une bouteille à la mer.

voix ex IMAGES Pour l'artiste que vous êtes avant tout, y a-t-il eu un déchirement lorsqu'on vous a proposé le poste de lieutenant-gouverneur de la province? Concilier ces deux rôles a-t-il été difficile pour vous? Cela a-t-il exigé une longue réflexion ou allait-il de soi que, pour la culture et l'avancement, cela valait la peine?

HERMÉnÉGILDE CHIASSON Toute ma vie, j'ai été impliqué dans la défense de la culture acadienne, j'ai fait partie de plusieurs associations, j'ai été président de l'Association des artistes acadiens et du Centre culturel Aberdeen pendant sept ans. Je me suis toujours vu comme un guerrier. Quand on m'a proposé ce poste, au départ, cela ne m'intéressait pas véritablement, et ensuite, j'ai été surpris qu'on ait pensé à moi, car le secteur culturel est toujours marginalisé par rapport à ce genre de nomination. Cela a changé ma vie, mais j'ai quand même continué mon activité artistique, de façon plus modérée. J'ai fait de la culture la grande priorité de mon mandat et je crois que le milieu culturel apprécie beaucoup cet engagement de ma part.

VoIX ET IMAGES Le côté conformiste et symbolique du poste soulevait-il des difficultés?

HERMÉnÉGILDE CHIASSON Cette dimension, c'est un peu du théâtre. Chaque fois que je me retrouve dans des situations très protocolaires, je me dis: "C'est du théâtre.» Mais il y a plein d'autres situations plus modestes ou courantes. Autrement, je m'arrange pour mettre davantage l'accent sur la dimension culturelle. Les trois priorités de mon mandat sont l'alphabétisation - au Nouveau-Brunswick, c'est vraiment un drame, surtout dans les régions acadiennes - , la culture, évidemment, puis les premières nations. Ces trois domaines sont finalement tous reliés à la culture.

voIX ET IMAGES Vous affirmiez l'an dernier dans L'Actualité que les enseignants en histoire au Nouveau-Brunswick devraient promouvoir une vision positive de l'histoire acadienne. Que vouliez-vous dire?

HERMÉNÉGILDE CHIASSON Je pense que la Déportation est devenue l'événement central de l'Acadie. J'ai souvent dit que c'est l'an 1 du peuple acadien. C'est à partir de ce moment-là que les Acadiens se sont vus comme un peuple. Mais il me semble 
qu'on insiste beaucoup sur un drame qui, souvent, engendre une colère sans réponse. Paradoxalement, tu entres dans l'Histoire au moment où tu en disparais. Trop d'éléments sont obnubilés. Je trouve que c'est vraiment important à l'heure actuelle de promouvoir une vision plus complète et surtout plus positive, plutôt que de se réduire à ce seul événement, cette immense défaite historique. Si on est là, c'est qu'il y a sûrement eu une résistance dont on entend peu sinon pas parler. Il y a eu des gens qui se sont sauvés, qui ont eu un courage énorme, admirable... Il faut aussi mettre l'accent sur la reconstruction, sur la manière dont les Acadiens sont arrivés à reprendre du pouvoir au lieu de tomber toujours dans un rapport de bourreauvictime qui finalement ne va nulle part.

VoIX ET IMAGES Vous redevenez artiste à temps plein après un mandat de six ans comme lieutenant-gouverneur du Nouveau-Brunswick. Quels sont les projets les plus pressants que vous voulez accomplir?

HERMÉNÉGILDE CHIASSON Je veux terminer ce roman, cette trilogie. Je voudrais aussi travailler au cinéma. J'ai plusieurs manuscrits que je voudrais publier et j'aimerais travailler au thêâtre en m'impliquant peut-être de manière plus directe, car jusqu'à maintenant, mon implication s'est souvent limitée à écrire un texte pour le donner à un metteur en scène... J'aimerais peut-être travailler de plus près à la production, faire des œuvres qui sont un peu plus organiques que la chaîne de production ordinaire du théâtre.

VoIX ET IMAGES Est-ce que le fait d'avoir franchi le cap de la soixantaine a changé quelque chose à la conception de votre travail artistique et de votre «mission" en Acadie? Qu'est-ce que vous aimeriez laisser au monde et à l'Acadie?

HERMÉNÉGILDE CHIASSON Cela a bien sûr changé des choses parce qu'on se rend compte que le temps est limité et qu'il y a un certain nombre de travaux qu'on veut faire, et qu'il faut les faire maintenant. Ce que j'aimerais laisser à l'Acadie, c'est une chronique, étant donné que j'aurai écrit sur une assez longue période, que j'aurai participé aux débats sociaux qui auront laissé des traces. Je pense qu'à ce momentlà, on pourra peut-être reconstituer un point de vue, une vision. Mon seul regret, c'est que j'aurais aimé qu'il y en ait plus, qu'on soit un plus grand nombre, mais je pense qu'il y a tout de même eu une grande amélioration dans les conditions de création comparativement à la génération qui m’a précédé. Cela étant dit, je suis toujours habité par ce désir latent qui fait qu'on peut continuer à vivre, à écrire, à faire des livres. 